\title{
The Display of Three-dimensional Anatomy With Stereolithographic Models
}

\author{
Nicholas J. Mankovich, Andrew M. Cheeseman, and Noel G. Stoker
}

Stereolithography, a new technique of prototype fabrication developed for the aerospace industry. offers a unique way to display patient anatomy. Like current computer aided design (CAD) systems, it uses digital image data from computed tomography (CT) and magnetic resonance (MR) to produce a physical model. Unlike conventional CAD it does not require a cutting tool and, therefore, CAD toolpath limitations do not exist. The stereolithography apparatus uses an ultraviolet laser to selectively polymerize and solidify a polymeric liquid plastic solution under computer control. The device was used to produce a model of cranial bony anatomy from CT image data, providing full internal detail in the constructed model, including encased sinuses, foramen, and potentially complete internal anatomy within a closed skull. The advantages and disadvantages of this technology are reviewed with an emphasis on future development.

(C) 1990 by Society of Photo-optical Instrumentation Engineers.

KEY WORDS: three-dimensional imaging, image display, computed tomography, computer aided design

QTEREOLITHOGRAPHY, a new technique $\circlearrowleft$ of prototype fabrication developed for the aerospace industry, offers a unique way to display patient anatomy. Like current medical computer aided manufacturing (CAM) systems, ${ }^{1-4}$ it can use digital image data from a tomographic modality to produce a physical model. Conventional CAM is a removal process employing a cutting tool to produce a model by cutting away material from a solid block. Stereolithography, on the other hand, is a constructive process producing a model by building it up layer by layer with plastic using an ultraviolet laser to catalyze the polymerization of a liquid plastic solution. ${ }^{6}$ By avoiding the tool path problems inherent in conventional CAM, stereolithogra-

From the Department of Radiological Sciences, University of California, Los Angeles, and Valley Medical Center Dental Clinic, Fresno, CA.

Address reprint requests to Nicholas J. Mankovich, Department of Radiological Sciences, Olive View-UCLA Medical Center, 14445 Olive View Dr, Sylmar, CA 91342.

(c) 1990 by Society of Photo-optical Instrumentation Engineers.

0897-1889/90/0303-0001\$03.00/0 phy allows full detail to be built into complex structures. This technology shows great promise for the construction of anatomic models from patient data and this paper reviews the basic techniques of stereolithography and reports on early efforts in medical model construction.

\section{MATERIALS AND METHODS}

Figure 1 shows the basic components involved in creating a model with the stereolithography apparatus (SLA). As normally used, the designer of an SLA model employs a computer aided design (CAD) system. These systems create objects as mathematical constructs and renders them for display on a CAD computer as either wireframe tracings or as surface shaded solids. In preparing data for SLA model construction, the data are represented as simple surface patch triangles that are transferred into the SLA Slice Computer (80386 personal computer) via network or magnetic tape. The Slice Computer transforms the surface triangle representation into a laser-compatible vector form by slicing this CAD representation at a user-specified interval. This slice vector data is then formatted to create a laser path instruction set which is downloaded to the SLA Control Computer. The SLA is composed of a controlling computer, an ultraviolet laser with optics and galvanometer-coupled mirrors, a liquid polymer tank, and a movable part platform within the tank.

Model fabrication starts with the tank full of liquid plastic and the data in the SLA Control Computer. The first layer of the model is created when the platform is lowered into the liquid and raised to a level just below the surface of the viscous liquid. The $10-\mathrm{mW}$ HeCd $(325-\mathrm{nm})$ laser, combined with a shutter and two computer-controlled galvanometer mirrors, draws the first slice and any supporting structures on the surface of the liquid. Wherever the laser strikes the surface of the liquid, the plastic solidifies into a bead .020 inch deep and .010 inch wide. By properly controlling the platform movement, liquid viscosity, and position of the laser, the solid plastic ridge adheres to the platform. After the layer is fully drawn, the platform and newly solidified layer are submerged in the liquid and then raised enough to guarantee another .020 inch layer of liquid on the previously solidified plastic. The next layer is then traced with the laser and the dipping process repeated. In this manner the solid model is built up of a succession of .020 inch layers. Wherever a surface in the original model is nearly horizontal, the computer fills in the region by drawing an overlapping hatched pattern to provide a solid segment. Typically, the SLA-1 can fabricate a completed piece in 8 hours of machine time. The piece is then moved to a curing oven where it is bathed in ultraviolet light for 10 minutes to complete model curing.

Stereolithography has found applications in the aerospace and automotive industries in the prototyping of computerdesigned parts. It has allowed the designer to create and view designs without recourse to more expensive and time consuming CAM fabrication processes. To a certain extent it has 
Fig 1. Basic components of the SLA and CT image data processing.

allowed the CAD shop to have its own three-dimensional (3D) hardcopy while allowing a type of prototyping not previously possible. Conventional CAM methods are limited by the size, shape, and speed of the cutting tools and the problems associated with orientation and fixation of the material to be cut. The SLA, a constructive process, does not have the orientation, depth, and undercutting problems encountered when trying to position a cutting bit around a complex surface. The SLA creates internal structures just as easily as it creates external structures. Linear shrinkage in SLA models is $1.2 \%$ in the xy plane and $0.2 \%$ along $\mathrm{z}$.

\section{CASE STUDY}

In order to establish the applicability of the stereolithography technology to medical model construction, a case was selected of a 20-year-old male with severe congenital craniofacial defects. This patient underwent surgery in which the maxilla was repositioned forward and stabilized with bone grafts (Modified LeFort III osteotomy) and the mandible was repositioned posteriorly with sliding osteotomies. After surgery, the patient was scanned for evaluation. The CT scan was done on a GE9800 at $120 \mathrm{kV}$ and $120 \mathrm{mAs}$ with $1.5 \mathrm{~mm}$ slice thickness at a $2.0 \mathrm{~mm}$ slice interval. Sixty-nine slices were produced and transferred to a VAX-11/750 (Digital Equipment, Maynard, MA) minicomputer and a PIXAR. 3D surface and volumetric reconstructions were done on the PIXAR using the Philips MSP (Philips Medical Systems, Shelton, CT) software package. The VAX computer was used to process the images for stereolithography.

CT slice processing traced all bone boundaries in the image volume after empirically determining the threshold for cortical bone. The algorithm exhaustively searched each image and located all of the inner and outer edges of cortical bone objects. The complete contour volume dataset was then passed to a reformat program which generated the SLA Build File containing the vectorized information necessary to guide the laser. During contour processing, each contour was replicated four times in order to provide a finished layer $2 \mathrm{~mm}$ thick. This repetition was necessary to reconstruct the CT axial resolution using the .020 in SLA layer thickness. In going from contour data to SLA data, inner and outer contours were filled with a honeycomb of vectors to provide near-solid plastic where solid bone existed in the CT images. Because the model was built of solid bone components layer by layer, it was not necessary to deal with the near-horizontal region filling encountered when dealing with triangulated data.

Once the SLA Build File was completed on the VAX, the data were written via network to a floppy disk. The disk data

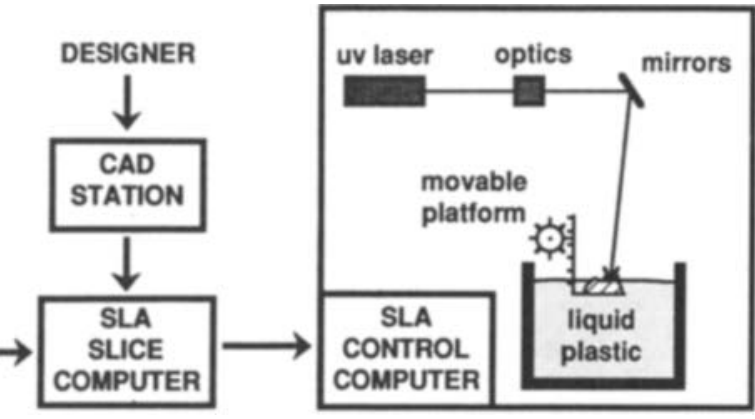

were then transferred to a Silicon Graphics (Mountain View, CA) IRIS workstation at 3D Systems, Incorporated (Valencia, CA). The data were read into the SLA graphics processing system and the Build File reviewed layer by layer. The SLA designer chose to construct the model from the top down, relying on the calvarium to provide a foundation for the smaller structures of the skull base. To support the first 0.5 in of construction of the model, a thin 0.5 in lattice was constructed and later merged onto the first 6 CT layers. Once this final CAD was complete, the SLA Build File (containing over 1.5 million vectors) was downloaded to the SLA Control Computer via network. Production of the model took 16 hours using the first-generation SLA-1 machine. After curing, the model was cleaned and the support lattice removed manually. Figure 2 shows the constructed model.

\section{DISCUSSION}

Stereolithography offers a new way of producing models of patient anatomy without the restrictions inherent in conventional CAD systems. We have successfully produced two models in this manner and have plans to further develop this technology. Overall, the process shows the promise of automatic model construction directly from CT images without the need for extensive operator interaction. This first model production identified areas requiring further development, specifically in image processing and the SLA process.

The ideal system should take CT images directly from a computer network, process the images, and then directly pass them onto the SLA Control Computer. We are in the process of porting our imaging software over to a Silicon Graphics Personal Iris 3D workstation. This 10-20 million instructions per second graphics computer will allow us to perform rapid image processing while integrating the vectorized edge output with the 3D System's CAD tools. Image processing will incorporate image interpolation ${ }^{6}$ to provide a more continuous surface on the model, down to the .020 in SLA resolution.

Our preliminary method relied on boundary following algorithms to produce the vectorized 

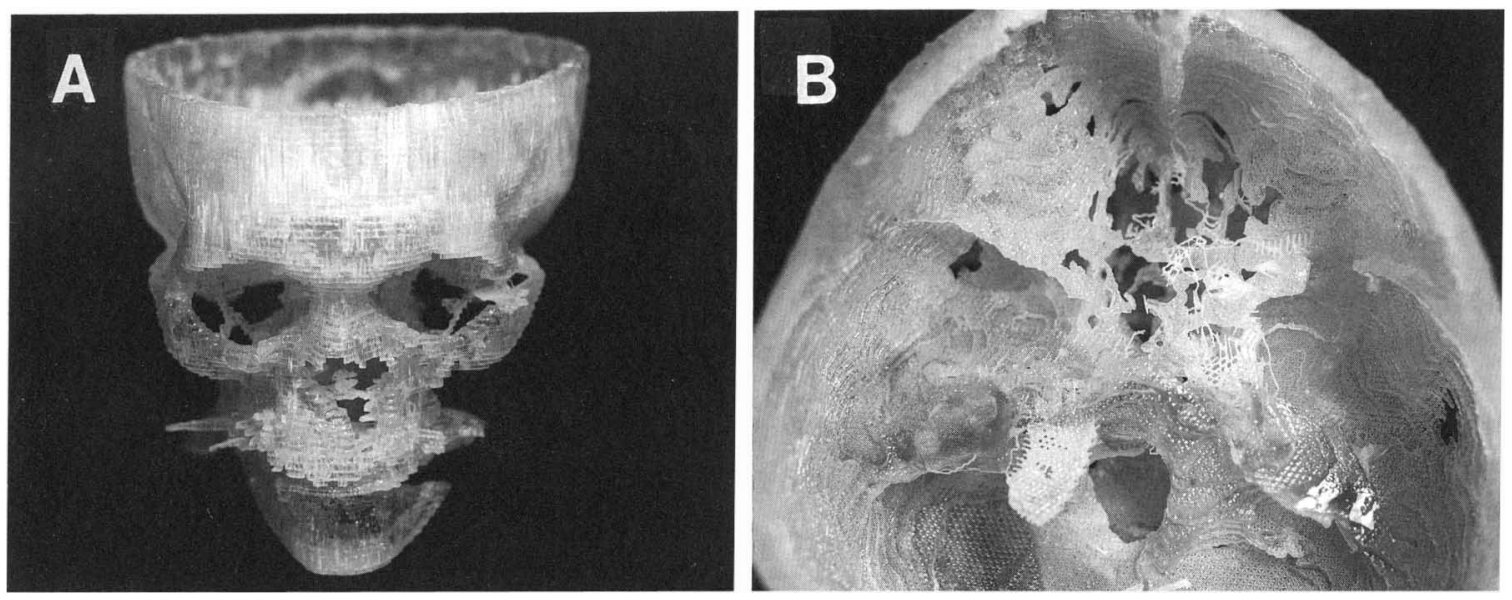

Fig 2. (A) Stereolithography skull model constructed from CT scan processing. Note the cleft upper palate and CT metal star artifact around the teeth. (B) Detail of the anterior portion of the skull base showing problems with floating contours (se日 text).

representation of bone edges, thus converting the raster images into a vector laser path. Where there is no need to manipulate the anatomical data, we hope that by properly processing the raster data and taking into account the lower level interfaces to the SLA, we can skip the vectorization and directly use the raster image data. This should reduce the processing to interpolation and thresholding.

Regardless of the method used to produce laser path data, the presence of floating contours presents a real problem. The floating contour condition occurs whenever new contours appear in an area which had no previous structure. Thus, the laser paints the new contour and it solidifies without any underlying structure, floating free in the plastic bath. To solve this problem, algorithms are being developed that simulate model creation, note when floating contours exist, and anchor the contours to an existing structure with a buttress. This simulation and correction process is similar to the tool path processing that is used with conventional numerically controlled machine tools.

The improvements to the SLA are part of its ongoing product development and include an improved data interchange format which is concise and verifiable prior to model construction. The introduction of the second generation SLA250 model doubles the speed of model creation and improves the performance of process software. One of the most serious concerns in the production of anatomic models is the accuracy with which the final product represents the original patient structure. Preliminary measurements of the model made to date show that the error in the $\mathrm{z}$ axis is less than $6 \%$ and is comparable to our previous method of using stacked $2 \mathrm{~mm}$ plastic cutouts. A more serious concern is model warpage introduced by differential shrinkage in the finished piece.

At present we are engaged in a project to determine the precision of the entire process from contour extraction to model fabrication. On the contour extraction side, we are working with a bone/soft tissue phantom and are using a variety of CT scanners to generate image data for contour extraction. The known dimensions of the phantom will be compared against those produced by various contouring algorithms applied to CT images. To test the SLA device, the phantom contour images will be used to construct an SLA model. This model will be measured immediately after creation and at regular intervals thereafter to determine the precision of the process and the dimensional stability of the model.

\section{CONCLUSION}

Stereolithography offers a new method for the production of anatomical models which promises to show full internal detail not previously attainable. The usefulness of such models extends to surgical planning, radiation therapy, patient education, and physician education. The remaining 
problems in stereolithography relate to the development of new algorithms and materials for model making. Solutions for these problems are forthcoming as we further investigate the process in the clinical setting.

\section{ACKNOWLEDGMENT}

The authors would like to thank the Department of Radiological Sciences Image Processing Laboratory for providing computational support in the processing of CT images. SLA design assistance by Scott Turner of 3-D Systems is greatly appreciated as is Dr Stuart White's help in photographic preparation.

\section{REFERENCES}

1. Dev P, Fellingham LL, Vassiliadis A, et al: 3D graphics for interactive surgical simulation and implant design. Process- ing and Display of Three-Dimensional Data II. Proc SPIE 507:52-57, 1984

2. Rothman SL, Glenn W, Rhodes M, et al: Individualized prosthesis production from routine CT data. Radiol $157: 177,1985$

3. Woolson ST, Dev P, Fellingham LL, et al: Threedimensional imaging of bone from computerized tomography. Clin Orthop 202:239-248, 1986

4. Robertson DD, Walker PS, Granholm JW, et al: Design of custom hip stem prostheses using three-dimensional CT modeling. J Comput Assist Tomogr 11:804-809, 1987

5. Wittenberg RC: CAD database creates solid models. Electronic Engineering Times 424:1, 1987

6. Heyers V, Dengler J, Meinzer HP: Approximation of missing sections of CT-image sequences using binary interpolation, in Magnenant-Thalman, Thalman (eds) New Trends in Computer Graphics. Berlin, FRG, Springer-Verlag, 1988, pp 537-545 